\title{
A uniform version of Laplace's method for contour integrals
}

\author{
Thorsten Neuschel
}

Received: October 10, 2011

Summary: It is the main objective of this note to establish a "simple and general" version of Laplace's method for obtaining approximations for complex contour integrals of the type $\int_{\mathcal{P}} e^{-n p(t, z)} q(t, z) \mathrm{d} t$, as $n \rightarrow \infty$, holding uniformly with respect to a parameter $z \in S$, where $S$ denotes some compact subset of $\mathbb{C}$. Moreover, two applications of this version are presented for illustration.

\section{Introduction}

Laplace's method is one of the classical procedures in asymptotic analysis for obtaining approximations of parameter integrals (see, e.g., [7, pp. 121-127]). These are complex contour integrals of the type

$$
\int_{\mathcal{P}} e^{-n p(t)} q(t) \mathrm{d} t
$$

where we consider the parameter $n$ to be a positive integer, $\mathcal{P}$ is a contour in the complex $t$-plane and the functions $p$ and $q$ are supposed to be holomorphic in a suitable domain. Although well-known versions of this method allow $n$ to be a complex quantity tending to infinity along an axis or even in an angular region here and in the sequel we deal with the integer case for $n$ only which is no essential restriction. In many applications of Laplace's method the integrals in (1.1) actually are of the type

$$
F_{n}(z)=\int_{\mathcal{P}} e^{-n p(t, z)} q(t, z) \mathrm{d} t
$$

now the functions $p$ and $q$ being continuous with respect to the pair of complex variables $(t, z)$ varying in some subset $G \times S \subset \mathbb{C}^{2}$ and for the fixed parameter $z$ the functions $p(\cdot, z)$, $q(\cdot, z)$ are holomorphic on the complex domain $G$. Applying Laplace's method mentioned above to the integral in (1.2) produces an asymptotic expansion for $F_{n}(z)$ as $n \rightarrow \infty$ which

AMS 2000 subject classification: Primary: 41A60; Secondary: 30E15

Key words and phrases: Approximation of parameter integrals, complete asymptotic expansion, Laplace's method 
holds pointwise for $z \in S$ for the time being. However, there are many instances for which a uniform validity of this expansion with respect to $z \in S$ is needed. As examples many sequences of polynomials may serve which possess an integral representation of type (1.2) such as the classical orthogonal polynomials (e.g. [11]) or more general many sequences of functions generated by a Rodrigues formula. In many papers dealing with asymptotics for special functions $F_{n}(z)$ an appropriate uniformity statement often is discussed by ad hoc arguments only. On the other hand, several authors have contributed to this subject from a general perspective, as, for instance, A. Erdélyi and M. Wyman (see [2]) and $\mathrm{N}$. Temme (see [12]). Relevant books as the standard monographs by Wong [13] and Olver [7] for example give a general description for obtaining asymptotic expansions for integrals (1.2) which are valid uniformly with respect to $z \in S$. In particular the aspect of uniformity usually is studied in connection with saddle points near an endpoint of the integration contour $\mathcal{P}$ or near a singularity of the integrand. Moreover, it is studied in the context of coalescing saddle points (e.g. [13, p. 353]). However, concerning more basic situations, as far as the author is aware there is no "simple and general" theorem for functions $F_{n}(z)$ in (1.2) giving a complete asymptotic expansion as $n \rightarrow \infty$ and holding uniformly with respect to $z \in S$. It is the main objective of this note to establish such a supplement to the well-known classical version for the integrals in (1.1) (see Theorem 3.1 below).

The last section contains two applications to well-known examples for illustration. A more elaborate application is treated in a forthcoming paper (see [5]) where the uniform version of Laplace's method is used for an asymptotic study of a certain class of generalized hypergeometric polynomials of type ${ }_{3} F_{1}$ which reveals, for instance, the asymptotic behavior of the ménage hit polynomials $U_{n}(t)$. These polynomials are given by

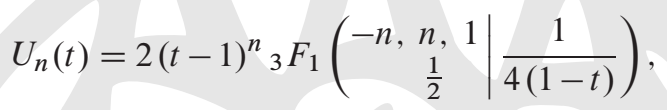

they arise in combinatorics in connection with the ménage problem and in modeling patterns on a chess board (see, e.g., [9, p. 197]).

\section{Auxiliary results}

In order to prove the main result in the next section we will state the two following lemmata. The first one can be considered as an easy generalization of the well-known fact that the zeros of a nonconstant holomorphic function defined on a domain are isolated (see, e.g. [10, p. 208]).

Lemma 2.1 Let $G \subset \mathbb{C}$ be a domain, $a \in G$, and $S \subset \mathbb{C}$ a nonempty compact set equipped with the subspace topology. Let

$$
p: G \times S \rightarrow \mathbb{C}
$$

be a continuous function such that the mapping $p_{z}:=p(\cdot, z)$ is holomorphic on $G$ for every $z \in S$. Suppose further that $p_{z}(a)=0$ and $p_{z}^{\prime}(a):=\left.\frac{\mathrm{d}}{\mathrm{d} t} p_{z}(t)\right|_{t=a} \neq 0$ for all $z \in S$. 
Then we can find an $\epsilon>0$ such that

$$
p_{z}(t) \neq 0
$$

for all $t \in \mathcal{U}_{\epsilon}(a) \backslash\{a\}$ and all $z \in S$ (here and in the sequel we define $\mathcal{U}_{\epsilon}(a):=\{t \in \mathbb{C}$ : $|t-a|<\epsilon\})$.

This lemma can be proved essentially by using slightly modified arguments from the proof of the classical result from complex function theory, so we will omit the details here (details can be found in [6, p. 20]).

Lemma 2.2 Under the assumptions of Lemma 2.1, we have the following statement: There is a $\delta>0$ and for every $z \in S$ there is an open connected neighborhood $B_{z} \subset G$ of the point a such that the mappings

$$
p_{z}: B_{z} \rightarrow \mathcal{U}_{\delta}(0)
$$

are conformal for all $z \in S$. Furthermore, the mappings

$$
q: \mathcal{U}_{\delta}(0) \times S \rightarrow \mathbb{C}, q(u, z):=p_{z}^{-1}(u)
$$

and

$$
q^{\prime}: \mathcal{U}_{\delta}(0) \times S \rightarrow \mathbb{C}, q^{\prime}(u, z):=\frac{\mathrm{d}}{\mathrm{d} u} q(u, z)
$$

are continuous. Moreover, there is an $\epsilon>0$ such that

$$
\mathcal{U}_{\epsilon}(a) \subset \bigcap_{z \in S} B_{z}
$$

and the point 0 is an interior point of the set

$$
\bigcap_{z \in S} p_{z}\left(\mathcal{U}_{\epsilon}(a)\right)
$$

\section{Proof:}

i) First we observe that the mapping

$$
p^{\prime}: G \times S \rightarrow \mathbb{C}, \quad p^{\prime}(t, z):=p_{z}^{\prime}(t):=\frac{\mathrm{d}}{\mathrm{d} t} p_{z}(t)
$$

is continuous, which can be obtained easily by using Cauchy's integral formula: For arbitrary $t_{0} \in G$ and $\delta_{1}>0$ such that $\overline{\mathcal{U}_{\delta_{1}}\left(t_{0}\right)} \subset G$ we have for $z \in S$ and $t \in \mathcal{U}_{\delta_{1}}\left(t_{0}\right)$

$$
p_{z}^{\prime}(t)=\frac{1}{2 \pi i} \oint_{\partial \mathcal{U}_{\delta_{1}}\left(t_{0}\right)} \frac{p_{z}(\zeta)}{(\zeta-t)^{2}} \mathrm{~d} \zeta
$$

hence we can deduce continuity by an application of standard theorems on continuity of parameter integrals. 
i i ) Let $\epsilon_{1}>0$ be a small positive number such that the following conditions hold:

a) $p_{z}(t) \neq 0$ for all $t \in \overline{\mathcal{U}_{\epsilon_{1}}(a)} \backslash\{a\}$ and for all $z \in S$ (this is possible according to Lemma 2.1).

$\beta) p_{z}^{\prime}(t) \neq 0$ for all $t \in \overline{\mathcal{U}_{\epsilon_{1}}(a)}$ and for all $z \in S$ (this is possible by virtue of the continuity of $p_{z}^{\prime}(t)$ and the assumed compactness of $S$ ).

Let the number $\delta>0$ be defined by

$$
\delta:=\min _{\substack{|t-a|=\epsilon_{1} \\ z \in S}}\left|p_{z}(t)\right|
$$

For arbitrary $z \in S$ and $w \in \mathcal{U}_{\delta}(0)$ the inequality

$$
\left|p_{z}(t)\right| \geq \delta>|w|
$$

holds for all $t$ lying on the circle $|t-a|=\epsilon_{1}$. Using Rouché's theorem we can deduce, that for all $z \in S$ and $w \in \mathcal{U}_{\delta}(0)$ the functions $p_{z}(t)$ and $p_{z}(t)-w$ have the same number of zeros inside $\mathcal{U}_{\epsilon_{1}}(a)$, which is according to condition $\alpha$ ) exactly one. This implies for all $z \in S$ the following statement:

For every $w \in \mathcal{U}_{\delta}(0)$ there is a unique $t \in \mathcal{U}_{\epsilon_{1}}(a)$ such that $p_{z}(t)=w$.

For $z \in S$ we define

$$
B_{z}:=p_{z}^{-1}\left(\mathcal{U}_{\delta}(0)\right) \cap \mathcal{U}_{\epsilon_{1}}(a)
$$

Then for every $z \in S$ the mapping

$$
p_{z}: B_{z} \rightarrow \mathcal{U}_{\delta}(0)
$$

is holomorphic and bijective, hence it is conformal according to a standard result from complex function theory. Moreover, for all $z \in S$ the set $B_{z}$ is a domain which clearly contains the point $a$.

i i i ) The claimed continuity of the mapping $q$ on $\mathcal{U}_{\delta}(0) \times S$ now can be established easily by using standard arguments exploiting the compactness of the set $\mathrm{S}$ (for details see [6, p. 23]). The continuity of $q^{\prime}$ then follows in a similar manner as the continuity of $p^{\prime}$ is shown in $i$ ).

iv) The existence of a small $\epsilon>0$ such that $\mathcal{U}_{\epsilon}(a) \subset \bigcap_{z \in S} B_{z}$ can be shown by proving that $a$ is an interior point of the set

$$
M:=\bigcap_{z \in S} q_{z}\left(\mathcal{U}_{\delta / 2}(0)\right)
$$

Suppose to the contrary, that $a$ is no interior point of $M$. Then we can find a sequence $\left(t_{n}\right)$ in $G$ such that $t_{n} \notin M$ for all $n \in \mathbb{N}$ and $t_{n} \rightarrow a, n \rightarrow \infty$. Hence, for every $n \in \mathbb{N}$ we can choose $z_{n} \in S$ such that $t_{n} \notin q_{z_{n}}\left(\mathcal{U}_{\delta / 2}(0)\right)$. As the statement $a \in q_{z}\left(\mathcal{U}_{\delta / 2}(0)\right)$ 
is valid for all $z \in S$, for every $n \in \mathbb{N}$ there is an intersection point $\tilde{t}_{n}$ of the boundary of $q_{z_{n}}\left(\mathcal{U}_{\delta / 2}(0)\right)$ and the straight line connecting the points $t_{n}$ and $a$. Consequently we have $\tilde{t}_{n} \rightarrow a$, as $n \rightarrow \infty$. Moreover, for every $\tilde{t}_{n}$ we can find $u_{n} \in \partial \mathcal{U}_{\delta / 2}(0)$ such that $q_{z_{n}}\left(u_{n}\right)=\tilde{t}_{n}$. Due to the compactness of $\partial \mathcal{U}_{\delta / 2}(0) \times S$, the sequence $\left(u_{n}, z_{n}\right)$ has a convergent subsequence $\left(u_{n_{k}}, z_{n_{k}}\right)$ with limit $\left(u_{0}, z_{0}\right) \in \partial \mathcal{U}_{\delta / 2}(0) \times S$. Using the continuity of $q$ we obtain

$$
q_{z_{0}}\left(u_{0}\right)=\lim _{k \rightarrow \infty} q_{z_{n_{k}}}\left(u_{n_{k}}\right)=\lim _{k \rightarrow \infty} \tilde{t}_{n_{k}}=a,
$$

which, in virtue of $q_{z_{0}}(0)=a$, contradicts the conformity of $q_{z_{0}}$ on $\mathcal{U}_{\delta}(0)$. Hence, $a$ is an interior point of $M$.

Finally, the fact that 0 is an interior point of $\bigcap_{z \in S} p_{z}\left(\mathcal{U}_{\epsilon}(a)\right)$ can be achieved in a similar manner.

\section{Main result}

The following uniform version of Laplace's method is based on the well-known classical procedure (see, e.g., [7, pp. 121-127]). We may assume, that the path of integration $\mathcal{P}$ is a piecewise continuously differentiable non-closed simple curve such that the derivative of a given parametrization on an open "interval of smoothness" always admits a continuous extension to the closure of this interval, which does not vanish there. We will adopt a convenient notation: Let $t_{1}$ and $t_{2}$ be any two points on the trace of $\mathcal{P}$, then we denote the part of the trace of $\mathcal{P}$ lying between $t_{1}$ and $t_{2}$ by $\left(t_{1}, t_{2}\right)_{\mathcal{P}}$ when $t_{1}$ and $t_{2}$ are both excluded, and by $\left[t_{1}, t_{2}\right]_{\mathcal{P}}$, when $t_{1}$ and $t_{2}$ are both included. Similarly for $\left(t_{1}, t_{2}\right]_{\mathcal{P}}$ and $\left[t_{1}, t_{2}\right)_{\mathcal{P}}$.

Theorem 3.1 Let $G \subset \mathbb{C}$ be a domain and $S \subset \mathbb{C}$ a nonempty compact set equipped with the subspace topology. Let $\mathcal{P}$ be a path, as defined above, with finite starting point $a \in G$ and finite or infinite endpoint $b$ such that $[a, b)_{\mathcal{P}} \subset G$. Let

$$
p, q: G \times S \rightarrow \mathbb{C}
$$

be continuous functions such that $p_{z}:=p(\cdot, z)$ and $q_{z}:=q(\cdot, z)$ are holomorphic on $G$ for all $z \in S$. In the neighborhood of $a$, for all $z \in S$ the functions $p_{z}$ and $q_{z}$ can be expanded in convergent series. Let these series be given by

$$
\begin{aligned}
& p_{z}(t)=p_{z}(a)+\sum_{\nu=0}^{\infty} b_{\nu}(z)(t-a)^{\mu+v}, \quad \mu \in \mathbb{N}, z \in S, b_{0}(z) \neq 0 \text { on } S, \\
& q_{z}(t)=\sum_{\nu=0}^{\infty} c_{\nu}(z)(t-a)^{\nu+\lambda-1}, \quad \lambda \in \mathbb{N}, z \in S, c_{0}(z) \neq 0 \text { on } S .
\end{aligned}
$$

Suppose, that for all $t \in(a, b)_{\mathcal{P}}$ the following condition holds

$$
\inf _{z \in S} \mathfrak{R}\left(p_{z}(t)-p_{z}(a)\right)>0,
$$

and, that there is a positive lower bound for this expression as $t \rightarrow b$ on $\mathcal{P}$. 
In addition suppose, that there is a number $N \in \mathbb{N}$ such that the complex contour integrals

$$
I(n, z):=\int_{\mathcal{P}} e^{-n p_{z}(t)} q_{z}(t) \mathrm{d} t
$$

converge absolutely for all $n \geq N$ and all $z \in S$, and that the expression

$$
\int_{\mathcal{P}} e^{-N \Re\left(p_{z}(t)\right)}\left|q_{z}(t)\right||\mathrm{d} t|
$$

is bounded when considered as a function of $z$ on $S$.

Then $I(n, z)$ possesses a complete asymptotic expansion of the form

$$
I(n, z)=\int_{\mathcal{P}} e^{-n p_{z}(t)} q_{z}(t) \mathrm{d} t \sim e^{-n p_{z}(a)} \sum_{\nu=0}^{\infty} \Gamma\left(\frac{\nu+\lambda}{\mu}\right) \frac{a_{\nu}(z)}{n^{(\nu+\lambda) / \mu}}, \quad n \rightarrow \infty .
$$

This expansion holds uniformly with respect to $z \in S$ and the first coefficients are given by

$$
a_{0}(z)=\frac{c_{0}(z)}{\mu\left(b_{0}(z)\right)^{\lambda / \mu}}, \quad a_{1}(z)=\left(\frac{c_{1}(z)}{\mu}-\frac{(\lambda+1) b_{1}(z) c_{0}(z)}{\mu^{2} b_{0}(z)}\right) \frac{1}{\left(b_{0}(z)\right)^{(\lambda+1) / \mu}} .
$$

(For the definition of the branches of powers with base $b_{0}(z)$ see the first part of the proof.)

\section{Proof:}

i) We begin by choosing a value of

$$
\omega:=\lim _{\substack{t \rightarrow a \\ t \in \mathcal{P}}} \arg (t-a)
$$

arbitrary but fixed (not necessary belonging to the interval $(-\pi, \pi]$ ). From the expansion for $p_{z}$ we obtain

$$
p_{z}(t)-p_{z}(a)=b_{0}(z)(t-a)^{\mu}\left(1+\sum_{\nu=1}^{\infty} \frac{b_{v}(z)}{b_{0}(z)}(t-a)^{v}\right) .
$$

Hence, by virtue of condition (3.1), for every $z \in S$ we can find a unique value of $\omega_{0}(z):=\arg b_{0}(z)$ such that

$$
\left|\omega_{0}(z)+\mu \omega\right| \leq \frac{\pi}{2}
$$

The continuity of the function $p$ implies the continuity of the functions $b_{0}$ and $\omega_{0}$ on the set $S$. In the sequel, all powers of $b_{0}(z)$ will be constructed using this branch, e.g.

$$
\left(b_{0}(z)\right)^{1 / \mu}:=\exp \left(\left(\log \left|b_{0}(z)\right|+i \omega_{0}(z)\right) / \mu\right) .
$$

Consequently, all those powers are continuous functions of $z$ on $S$. 
ii) For $z \in S$ and $t \in G$ we define

$$
v_{z}(t):=p_{z}(t)-p_{z}(a)
$$

Let the branch of $\arg \left(v_{z}(t)\right)$ be chosen such that it is continuous on $\mathcal{P}$ and

$$
\arg \left(v_{z}(t)\right) \rightarrow \omega_{0}(z)+\mu \omega, t \rightarrow a, t \in(a, b)_{\mathcal{P}},
$$

where we note that $v_{z}(t) \neq 0$ for all $z \in S$ and $t \in(a, b)_{\mathcal{P}}$. From condition (3.1), for all $t \in(a, b)_{\mathcal{P}}$ we obtain

$$
\sup _{z \in S}\left|\arg \left(v_{z}(t)\right)\right|<\frac{\pi}{2} .
$$

In the sequel all powers of $v_{z}(t)$ for $t \in(a, b)_{\mathcal{P}}$ and $z \in S$ will be constructed using this branch. From the continuity of $p$ on $G \times S$ we can deduce, by using Cauchy's integral formula, the continuity of the function

$$
\frac{p_{z}(t)-p_{z}(a)}{(t-a)^{\mu}}
$$

on $G \times S$, which clearly implies the continuity of the function

$$
g_{z}(t):=g(t, z):=\frac{1}{b_{0}(z)} \frac{p_{z}(t)-p_{z}(a)}{(t-a)^{\mu}}-1=\sum_{\nu=1}^{\infty} \frac{b_{v}(z)}{b_{0}(z)}(t-a)^{v}
$$

on $G \times S$. From $g_{z}(a)=0$ for all $z \in S$ follows, that there is a $\rho>0$ such that

$$
\sup _{\substack{z \in S \\ t \in \mathcal{U} \rho(a)}}\left|g_{z}(t)\right|<\frac{1}{2} \text {. }
$$

Let

$$
w(t, z):=\left(b_{0}(z)\right)^{1 / \mu}(t-a)\left(1+g_{z}(t)\right)^{1 / \mu}, t \in \mathcal{U}_{\rho}(a), \quad z \in S,
$$

where the expression $\left(1+g_{z}(t)\right)^{1 / \mu}$ is defined by choosing the principle branch of $x^{1 / \mu}$ on $\mathbb{C} \backslash(-\infty, 0]$ and evaluating it at $x=1+g_{z}(t)$. It follows, that the function

$$
w: \mathcal{U}_{\rho}(a) \times S \rightarrow \mathbb{C}
$$

is continuous, and $w_{z}:=w(\cdot, z)$ is holomorphic on $\mathcal{U}_{\rho}(a)$ for every $z \in S$. Furthermore, we observe

$$
w_{z}(a)=0,\left.\frac{\mathrm{d}}{\mathrm{d} t} w_{z}(t)\right|_{t=a}=\left(b_{0}(z)\right)^{1 / \mu} \neq 0
$$

on $S$, and we have

$$
\left(w_{z}(t)\right)^{\mu}=v_{z}(t)
$$

for $t \in \mathcal{U}_{\rho}(a)$ and $z \in S$. By applying Lemma 2.2 to the function $w$ we obtain:

There is a $\delta>0$ and there are open connected neighborhoods $B_{z} \subset \mathcal{U}_{\rho}(a)$ of the point $a$, such that the functions

$$
w_{z}: B_{z} \rightarrow \mathcal{U}_{\delta}(0)
$$


are conformal for all $z \in S$. Moreover, there is an $\epsilon>0$ such that

$$
\mathcal{U}_{\epsilon}(a) \subset \bigcap_{z \in S} B_{z}
$$

and

$$
\begin{aligned}
& h: \mathcal{U}_{\delta}(0) \times S \rightarrow \mathbb{C}, h_{z}(u):=h(u, z)=w_{z}^{-1}(u), \\
& h^{\prime}: \mathcal{U}_{\delta}(0) \times S \rightarrow \mathbb{C}, \quad h_{z}^{\prime}(u):=\frac{\mathrm{d}}{\mathrm{d} u} h(u, z)
\end{aligned}
$$

are continuous functions.

i i i ) Let $k \in(a, b)_{\mathcal{P}}$ be chosen sufficiently close to $a$ to ensure $[a, k]_{\mathcal{P}} \subset \mathcal{U}_{\epsilon}(a)$. Then we have for $z \in S$

$$
\mathcal{U}_{\left|w_{z}(k)\right|}(0) \subset \mathcal{U}_{\delta}(0),
$$

and from $w_{z}(k) \neq 0$ on $S$ follows

$$
\min _{z \in S}\left|w_{z}(k)\right|>0
$$

For every $z \in S$ the path $[a, k]_{\mathcal{P}}$ may be deformed to make its image $w_{z}\left([a, k]_{\mathcal{P}}\right)$ a straight line connecting the points 0 and $w_{z}(k)$. Using Cauchy's integral theorem we find $\left(t=h_{z}(s)\right)$

$$
\int_{[a, k]_{\mathcal{P}}} e^{-n p_{z}(t)} q_{z}(t) \mathrm{d} t=e^{-n p_{z}(a)} \int_{0}^{w_{z}(k)} e^{-n s^{\mu}} q_{z}\left(h_{z}(s)\right) h_{z}^{\prime}(s) \mathrm{d} s,
$$

where the path of integration in the last integral is given by a straight line. Moreover, the function defined by

$$
f: \mathcal{U}_{\delta}(0) \times S \rightarrow \mathbb{C}, \quad f_{z}(s):=f(s, z):=q_{z}\left(h_{z}(s)\right) h_{z}^{\prime}(s)
$$

is continuous and $f_{z}$ is holomorphic on $\mathcal{U}_{\delta}(0)$ for all $z \in S$. Hence, $f_{z}$ has a representation of the form

$$
f_{z}(s)=\sum_{\nu=0}^{\infty} d_{\nu}(z) s^{\nu+\lambda-1}, s \in \mathcal{U}_{\delta}(0), \lambda \in \mathbb{N} \text { fixed. }
$$

Consequently we find (using $s=u^{1 / \mu}$ in the second equality)

$$
\begin{aligned}
\int_{[a, k]_{\mathcal{P}}} e^{-n p_{z}(t)} q_{z}(t) \mathrm{d} t & =e^{-n p_{z}(a)} \int_{0}^{w_{z}(k)} e^{-n s^{\mu}} f_{z}(s) \mathrm{d} s \\
& =e^{-n p_{z}(a)} \int_{0}^{v_{z}(k)} e^{-n u} f_{z}\left(u^{1 / \mu}\right) \frac{1}{\mu} u^{1 / \mu-1} \mathrm{~d} u,
\end{aligned}
$$


where, again, the path of integration in the last integral is given by a straight line and for the expression $u^{1 / \mu}$ we choose the principle branch on $\mathbb{C} \backslash(-\infty, 0]$. Let $\tilde{\delta}:=\delta^{\mu}$, then the function

$$
\tilde{f}:\left(\mathcal{U}_{\tilde{\delta}}(0) \cap\{u \in \mathbb{C} \mid \Re(u)>0\}\right) \times S \rightarrow \mathbb{C}, \quad \tilde{f}(u, z):=\frac{1}{\mu} f_{z}\left(u^{1 / \mu}\right) u^{1 / \mu-1}
$$

can be represented by a series of the form

$$
\tilde{f}_{z}(u):=\tilde{f}(u, z)=\sum_{\nu=0}^{\infty} a_{v}(z) u^{(v+\lambda-\mu) / \mu},
$$

where the coefficients $a_{v}(z)$ can be derived from the coefficients $b_{v}(z)$ and $c_{v}(z)$. The first two coefficients may be verified to be

$$
a_{0}(z)=\frac{c_{0}(z)}{\mu\left(b_{0}(z)\right)^{\lambda / \mu}}, a_{1}(z)=\left(\frac{c_{1}(z)}{\mu}-\frac{(\lambda+1) b_{1}(z) c_{0}(z)}{\mu^{2} b_{0}(z)}\right) \frac{1}{\left(b_{0}(z)\right)^{(\lambda+1) / \mu}} .
$$

Furthermore, the coefficients $a_{v}(z)$ are related to those of $f$ by

$$
d_{v}(z)=\mu a_{v}(z)
$$

Now let $m \in \mathbb{N}_{0}$ and

$$
F_{m}(s, z):=\sum_{\nu=m}^{\infty} d_{\nu}(z) s^{\nu-m}, \quad s \in \mathcal{U}_{\delta}(0), \quad z \in S .
$$

Thus for $s \neq 0$ we obtain

$$
F_{m}(s, z)=s^{-m-\lambda+1}\left(f_{z}(s)-\sum_{\nu=0}^{m-1} d_{\nu}(z) s^{\nu+\lambda-1}\right),
$$

and $F_{m}(\cdot, z)$ is a holomorphic function of $s$ on $\mathcal{U}_{\delta}(0)$ for all $m \in \mathbb{N}_{0}$ and all $z \in S$. For every $m \in \mathbb{N}_{0}$ the continuity of $f$ on the set $\mathcal{U}_{\delta}(0) \times S$ implies, by using Cauchy's integral formula, the continuity of $F_{m}$, so that we can state the following:

For every $m \in \mathbb{N}_{0}$ the function $F_{m}$ is continuous on $\mathcal{U}_{\delta}(0) \times S$ and therefore it is bounded on every compact subset of $\mathcal{U}_{\delta}(0) \times S$.

Let $m \in \mathbb{N}_{0}$ and

$$
\tilde{F}_{m}(u, z):=\sum_{\nu=m}^{\infty} a_{\nu}(z) u^{(\nu-m) / \mu}, \quad z \in S, u \in \mathcal{U}_{\tilde{\delta}}(0) \cap\{u \in \mathbb{C} \mid \Re(u) \geq 0\},
$$

then we have $\tilde{F}_{m}(u, z)=\frac{1}{\mu} F_{m}\left(u^{1 / \mu}, z\right)$. Hence, for every $m \in \mathbb{N}_{0}$ the function $\tilde{F}_{m}$ is bounded on every compact subset of the set

$\left(\mathcal{U}_{\tilde{\delta}}(0) \cap\{u \in \mathbb{C} \mid \Re(u) \geq 0\}\right) \times S$. 
Moreover, we find

$$
\tilde{f}_{z}(u)=\sum_{\nu=0}^{m-1} a_{\nu}(z) u^{(\nu+\lambda-\mu) / \mu}+u^{(m+\lambda-\mu) / \mu} \tilde{F}_{m}(u, z)
$$

for $z \in S$ and $u \in \mathcal{U}_{\tilde{\delta}}(0) \cap\{u \in \mathbb{C} \mid \Re(u)>0\}$. Thus for $m \in \mathbb{N}_{0}$ and $z \in S$ we have

$$
\int_{0}^{v_{z}(k)} e^{-n u} \tilde{f}_{z}(u) \mathrm{d} u=\sum_{\nu=0}^{m-1} \Gamma\left(\frac{v+\lambda}{\mu}\right) \frac{a_{v}(z)}{n^{(v+\lambda) / \mu}}-\epsilon_{m, 1}(n, z)+\epsilon_{m, 2}(n, z),
$$

where, using the incomplete Gamma function, we set

$$
\begin{aligned}
\epsilon_{m, 1}(n, z) & :=\sum_{\nu=0}^{m-1} \Gamma\left(\frac{v+\lambda}{\mu}, v_{z}(k) n\right) \frac{a_{v}(z)}{n^{(v+\lambda) / \mu}}, \\
\epsilon_{m, 2}(n, z) & :=\int_{0}^{v_{z}(k)} e^{-n u} u^{(m+\lambda-\mu) / \mu} \tilde{F}_{m}(u, z) \mathrm{d} u .
\end{aligned}
$$

Because $\sup _{z \in S}\left|\arg \left(v_{z}(k)\right)\right|<\frac{\pi}{2}$, by applying an asymptotic result for the incomplete Gamma function (see, e.g. [7, p. 110]) we obtain

$$
\epsilon_{m, 1}(n, z)=\mathcal{O}\left(e^{-n v_{z}(k)} / n\right)=\mathcal{O}\left(e^{-n \eta} / n\right), n \rightarrow \infty,
$$

uniformly with respect to $z \in S$ for some $\eta$ satisfying $0<\eta \leq \Re\left(v_{z}(k)\right)$ on $S$. Moreover, we find $\left(u=v_{z}(k) \tau\right)$

$$
\begin{aligned}
\left|\epsilon_{m, 2}(n, z)\right| & =\left|\int_{0}^{1} e^{-n v_{z}(k) \tau}\left(v_{z}(k) \tau\right)^{(m+\lambda-\mu) / \mu} \tilde{F}_{m}\left(v_{z}(k) \tau, z\right) v_{z}(k) \mathrm{d} \tau\right| \\
& \leq \int_{0}^{1} e^{-n \Re\left(v_{z}(k)\right) \tau} \tau^{(m+\lambda-\mu) / \mu}\left|\left(v_{z}(k)\right)^{(m+\lambda) / \mu} \tilde{F}_{m}\left(v_{z}(k) \tau, z\right)\right| \mathrm{d} \tau .
\end{aligned}
$$

From the estimation

$$
\left|\left(v_{z}(k)\right)^{(m+\lambda) / \mu} \tilde{F}_{m}\left(v_{z}(k) \tau, z\right)\right| \leq K,
$$

where $K>0$ is a suitable constant independent of $z$, it follows (using $\tau=s / n \eta$ )

$$
\begin{aligned}
\left|\epsilon_{m, 2}(n, z)\right| & \leq K \int_{0}^{1} e^{-n \eta \tau} \tau^{(m+\lambda-\mu) / \mu} \mathrm{d} \tau \\
& =K \int_{0}^{n \eta} e^{-s} s^{(m+\lambda-\mu) / \mu}(n \eta)^{-(m+\lambda) / \mu} \mathrm{d} s \\
& =\mathcal{O}\left(\frac{1}{n^{(m+\lambda) / \mu}}\right), n \rightarrow \infty,
\end{aligned}
$$


uniformly with respect to $z \in S$. Altogether we obtain

$$
\begin{aligned}
& \int_{[a, k]_{\mathcal{P}}} e^{-n p_{z}(t)} q_{z}(t) \mathrm{d} t \\
& \quad=e^{-n p_{z}(a)}\left(\sum_{\nu=0}^{m-1} \Gamma\left(\frac{\nu+\lambda}{\mu}\right) \frac{a_{\nu}(z)}{n^{(v+\lambda) / \mu}}+\mathcal{O}\left(\frac{1}{n^{(m+\lambda) / \mu}}\right)\right),
\end{aligned}
$$

as $n \rightarrow \infty$, uniformly with respect to $z \in S$.

$i v$ ) From condition (3.1) we find some $\kappa>0$ such that

$$
\Re\left(p_{z}(t)-p_{z}(a)\right) \geq \kappa>0
$$

holds for all $z \in S$ and for all $t \in[k, b)_{\mathcal{P}}$. Consequently we have for all $n \geq N$

$$
\begin{aligned}
& \left|\int_{[k, b)_{\mathcal{P}}} e^{-n p_{z}(t)} q_{z}(t) \mathrm{d} t\right| \\
& \quad \leq\left|e^{-n p_{z}(a)}\right| e^{(N-n) \kappa} e^{N \Re\left(p_{z}(a)\right)} \int_{[k, b)_{\mathcal{P}}} e^{-N \Re\left(p_{z}(t)\right)}\left|q_{z}(t)\right||\mathrm{d} t| .
\end{aligned}
$$

It follows from the assumptions above, that the expression

$$
\int_{[k, b)_{\mathcal{P}}} e^{-N \Re\left(p_{z}(t)\right)}\left|q_{z}(t)\right||\mathrm{d} t|
$$

is bounded when considered as a function of $z$ on $S$. If $\tilde{K}$ is a suitable chosen constant, we have

$$
\left|\int_{[k, b)_{\mathcal{P}}} e^{-n p_{z}(t)} q_{z}(t) \mathrm{d} t\right| \leq \tilde{K}\left|e^{-n p_{z}(a)}\right| e^{-n \kappa},
$$

which implies

$$
\int_{[k, b)_{\mathcal{P}}} e^{-n p_{z}(t)} q_{z}(t) \mathrm{d} t=e^{-n p_{z}(t)} \mathcal{O}\left(e^{-n \kappa}\right), \quad n \rightarrow \infty,
$$

uniformly with respect to $z \in S$. Finally this completes the proof. 


\section{Applications}

In this section we will give two applications of Theorem 3.1 for illustration. The first one treats the formula of Laplace and Heine for Legendre polynomials (see, e.g. [11, p. 194] or for a more general statement see, e.g. [3, p. 117])

Example 4.1 Let $P_{n}$ denote the $n$-th Legendre polynomial. Then we have

$$
P_{n}(z)=\frac{\left(z^{2}-1\right)^{-1 / 4}}{\sqrt{2 \pi n}}\left(z+\sqrt{z^{2}-1}\right)^{n+1 / 2}\left(1+\mathcal{O}\left(\frac{1}{\sqrt{n}}\right)\right), \text { as } n \rightarrow \infty,
$$

uniformly on compact subsets of $\mathbb{C} \backslash[-1,1]$.

Proof: Let $S$ be a nonempty compact subset of $\mathbb{C} \backslash[-1,1] \cap\{z \in \mathbb{C} \mid \Re z \geq 0\}$. We can restrict our considerations to compact subsets of the right half-plane, because the remaining case can be settled in an similar manner, from which the conclusion readily follows. We represent $P_{n}$ by using the first integral of Laplace (see, e.g. [3, p. 96]):

$$
\begin{aligned}
P_{n}(z) & =\frac{1}{\pi} \int_{0}^{\pi}\left(z+\sqrt{z^{2}-1} \cos t\right)^{n} \mathrm{~d} t \\
& =\frac{1}{\pi} \int_{0}^{\epsilon} e^{-n p_{z}(t)} \mathrm{d} t+\frac{1}{\pi} \int_{\epsilon}^{\pi}\left(z+\sqrt{z^{2}-1} \cos t\right)^{n} \mathrm{~d} t,
\end{aligned}
$$

where $p_{z}(t):=-\log \left(z+\sqrt{z^{2}-1} \cos t\right), 0 \leq t<\epsilon, z \in S$, and $0<\epsilon<\pi$ chosen sufficiently small to ensure holomorphy of $p_{z}$ on $\mathcal{U}_{\epsilon}(0)$ for all $z \in S$. Here the branch of the logarithm is chosen to be the principle branch on $\mathbb{C} \backslash(-\infty, 0]$. We have

$$
p_{z}^{\prime}(t)=\frac{\sqrt{z^{2}-1} \sin t}{z+\sqrt{z^{2}-1} \cos t}=0, \text { when } t=0,
$$

and

$$
p_{z}^{\prime \prime}(0)=\frac{\sqrt{z^{2}-1}}{z+\sqrt{z^{2}-1}}, \quad z \in S .
$$

Furthermore, using $\left|z+\sqrt{z^{2}-1}\right|>1>\left|z-\sqrt{z^{2}-1}\right|$ we obtain

$$
\left|z+\sqrt{z^{2}-1}\right|>\left|z+\sqrt{z^{2}-1} \cos t\right|
$$

holding for all $z \in \mathbb{C} \backslash[-1,1]$ and $0<t \leq \pi$. Hence, we find

$$
\Re\left(p_{z}(t)-p_{z}(0)\right)=\log \left|\frac{z+\sqrt{z^{2}-1}}{z+\sqrt{z^{2}-1} \cos t}\right|>0
$$


holding for all $z \in S$ and $0<t \leq \epsilon$. Consequently, all assumptions of Theorem 3.1 clearly are satisfied and it follows that $(\mu=2, \lambda=1)$

$$
\frac{1}{\pi} \int_{0}^{\epsilon} e^{-n p_{z}(t)} \mathrm{d} t=\frac{\left(z^{2}-1\right)^{-1 / 4}}{\sqrt{2 \pi n}}\left(z+\sqrt{z^{2}-1}\right)^{n+1 / 2}\left(1+\mathcal{O}\left(\frac{1}{\sqrt{n}}\right)\right),
$$

as $n \rightarrow \infty$, uniformly with respect to $z \in S$. Moreover, using (4.2) we easily obtain

$$
\begin{aligned}
& \frac{1}{\pi} \int_{\epsilon}^{\pi}\left(z+\sqrt{z^{2}-1} \cos t\right)^{n} \mathrm{~d} t \\
& \quad=\frac{1}{\sqrt{2 \pi n}}\left(\frac{z+\sqrt{z^{2}-1}}{\sqrt{z^{2}-1}}\right)^{1 / 2}\left(z+\sqrt{z^{2}-1}\right)^{n} \mathcal{O}\left(\frac{1}{\sqrt{n}}\right),
\end{aligned}
$$

as $n \rightarrow \infty$, holding uniformly with respect to $z \in S$. Altogether, in virtue of (4.1), this establishes the statement.

In the second example we deal with the horizontal generating functions $T_{n}$ of the Stirling numbers of the second kind $S(n, k)$, which occasionally are termed as Touchard polynomials. These polynomials are given by

$$
T_{n}(z)=\sum_{k=1}^{n} S(n, k) z^{k}
$$

and they can be represented by

$$
T_{n}(z)=\frac{n !}{2 \pi i} \int_{\gamma_{0}} \frac{\exp \left\{z\left(e^{w}-1\right)\right\}}{w^{n+1}} \mathrm{~d} w,
$$

where the path of integration is given by a positive oriented circle enclosing the origin. In the paper [1] an asymptotic formula for the normalized sequence $T_{n}(n z)$ is proven, which is valid uniformly on compact subsets of $\mathbb{C} \backslash[-e, 0]$. In doing so, the uniformity is established by ad hoc arguments, whereas we will achieve it by an application of Theorem 3.1. This actually is an illustrative example of establishing uniformity of asymptotics which are obtained by use of the saddle point method.

For convenience, we define a mapping $\Phi: \mathbb{C} \backslash[-e, 0] \rightarrow \mathbb{C}$ by $\Phi(z)=W(1 / z)$, where $W$ denotes the principle branch of the LambertW function (see, e.g., [8, p. 111]). It is clear from [4, pp. 346-350], that $\Phi$ maps $\mathbb{C} \backslash[-e, 0]$ conformally onto a set $\mathcal{A}$ which is given by

$$
\mathcal{A}:=\{w=a+i b \in \mathbb{C} \mid a+1>0,-\pi<b<\pi \text { and } a+b \cot b>0\} .
$$

Example 4.2 (See [1, pp. 209, 211].) Let $T_{n}$ denote the $n$-th Touchard polynomial. Then

$$
T_{n}(n z)=\frac{n !}{\sqrt{2 \pi n}} \Phi(z)^{-n} \exp \left\{\frac{n}{\Phi(z)}\left(1-e^{-\Phi(z)}\right)\right\}(1+\Phi(z))^{-1 / 2}\left(1+\mathcal{O}\left(\frac{1}{n}\right)\right),
$$

as $n \rightarrow \infty$, holding uniformly on compact subsets of $\mathbb{C} \backslash[-e, 0]$. 
Proof: Let $S$ be a nonempty compact subset of $\mathbb{C} \backslash[-e, 0]$. In the representation (4.3) we choose $\gamma_{0}(t)=\Phi(z) e^{i t},-\pi<t<\pi$, and we find (after splitting the integral in two parts)

$$
T_{n}(n z)=\frac{n !}{\sqrt{2 \pi n}} \Phi(z)^{-n}\left\{\int_{0}^{\pi} e^{-n p_{z}(t)} \mathrm{d} t+\int_{0}^{\pi} e^{-n p_{z}(-t)} \mathrm{d} t\right\},
$$

where $p_{z}(t):=i t-z\left(\exp \left\{\Phi(z) e^{i t}\right\}-1\right), t \in \mathbb{C}, z \in S$. From [1, pp. 206-209], we know $\Re\left(p_{z}(t)-p_{z}(0)\right)>0$ for $t \in(-\pi, \pi) \backslash\{0\}$ and $z \in S$, which, together with the fact that $\Phi(z)$ is a simple saddle point, gives reason for the choice of $\gamma_{0}$. Therefore, the assumptions of Theorem 3.1 are easily seen to be satisfied for both integrals in (4.4) and we obtain asymptotics of the form ( $\mu=2$ and $\lambda=1$ there)

$$
\begin{aligned}
& \int_{0}^{\pi} e^{-n p_{z}(t)} \mathrm{d} t=e^{-n p_{z}(0)}\left\{\frac{\sqrt{\pi} a_{0}(z)}{\sqrt{n}}+\frac{a_{1}(z)}{n}+\mathcal{O}\left(\frac{1}{n^{3 / 2}}\right)\right\}, \\
& \int_{0}^{\pi} e^{-n p_{z}(-t)} \mathrm{d} t=e^{-n p_{z}(0)}\left\{\frac{\sqrt{\pi} a_{0}(z)}{\sqrt{n}}-\frac{a_{1}(z)}{n}+\mathcal{O}\left(\frac{1}{n^{3 / 2}}\right)\right\},
\end{aligned}
$$

as $n \rightarrow \infty$, holding uniformly with respect to $z \in S$. Hence, we find

$$
\int_{0}^{\pi} e^{-n p_{z}(t)} \mathrm{d} t+\int_{0}^{\pi} e^{-n p_{z}(-t)} \mathrm{d} t=e^{-n p_{z}(0)}\left\{\frac{2 \sqrt{\pi} a_{0}(z)}{\sqrt{n}}+\mathcal{O}\left(\frac{1}{n^{3 / 2}}\right)\right\},
$$

as $n \rightarrow \infty$, holding uniformly with respect to $z \in S$. Using the identity $\Phi(z) e^{\Phi(z)}=1 / z$, it can be verified that

$$
\begin{aligned}
& p_{z}(0)=-z\left(e^{\Phi(z)}-1\right)=-\frac{1-e^{-\Phi(z)}}{\Phi(z)}, \\
& a_{0}(z)=\frac{1}{\sqrt{2}}(1+\Phi(z))^{-1 / 2},
\end{aligned}
$$

from which the statement follows.

A more elaborate application of Theorem 3.1 is treated in the paper [5].

\section{References}

[1] C. Elbert. Strong asymptotics of the generating polynomials of the Stirling numbers of the second kind. J. Approx. Theory, 109:198-217, 2001.

[2] A. Erdélyi, M. Wyman. The asymptotic evaluation of certain integrals. Arch. Rational Mech. Anal., 14:217-260, 1963. 
[3] M. Ismail. Classical and Quantum Orthogonal Polynomials in One Variable. Cambridge University Press, 2005.

[4] D. Lubinsky, A. Sidi. Strong asymptotics for polynomials biorthogonal to powers of $\log x$. Analysis, 14:341-379, 1994.

[5] T. Neuschel. Asymptotics for Ménage polynomials and certain hypergeometric polynomials of type ${ }_{3} F_{1}$. J. Approx. Theory, 164:981-1006, 2012.

[6] T. Neuschel. Asymptotiken für Ménage-Polynome. Dissertation, University of Trier, 2011.

[7] F. Olver. Asymptotics and Special Functions. Academic Press, 1974.

[8] F. Olver, D. Lozier, R. Boisvert, C. Clark. NIST Handbook of Mathematical Functions. Cambridge University Press, 2010.

[9] J. Riordan. An Introduction to Combinatorial Analysis. J. Wiley \& Sons, fourth edition, 1967.

[10] W. Rudin. Real and Complex Analysis. McGraw-Hill, third edition, 1987.

[11] G. Szegö. Orthogonal Polynomials. American Mathematical Society, fourth edition, 1975.

[12] N. Temme. Uniform asymptotic expansions of Laplace integrals. Analysis, 3:221249, 1983.

[13] R. Wong. Asymptotic Approximation of Integrals. Society for Industrial Mathematics, 2001.

Thorsten Neuschel

Department of Mathematics

University of Trier

D-54286 Trier

Germany

neuschel@uni-trier.de 